\title{
Approche variationnelle de l'endommagement : II. Les modèles à gradient
}

\author{
Kim Pham ${ }^{\text {a Jean-Jacques Marigo }}{ }^{\mathrm{b}}$ \\ anstitut Jean le Rond d'Alembert (UMR 7190), Université Paris VI \\ ${ }^{\mathrm{b}}$ Laboratoire de Mécanique des Solides (UMR 7649), École Polytechnique
}

\begin{abstract}
Résumé
Cette deuxième partie de l'approche variationnelle de l'endommagement est consacrée à la construction de modèles non locaux, à gradient d'endommagement. Elle consiste à étendre à ces modèles régularisés les concepts introduits dans la première partie dans le cadre de modèles locaux. Une fois que les termes à gradient d'endommagement ont été introduits dans l'énergie, le problème d'évolution s'écrit toujours à partir des trois principes physiques d'irréversibilité, de stabilité et de bilan d'énergie. Cette nouvelle formulation est comparée à celle usuellement proposée, mettant en évidence ses apports.
\end{abstract}

Abstract. The variational approach to damage : II. The gradient damage models. This second part of the variational approach to damage is devoted to the construction of non local gradient-enhanced models. That consists in extending to these regularized models the concepts introduced in the first part for local damage models. Specifically, once the gradient of damage has been inserted into the energy expression, the damage evolution problem is still based on the three physical principles of irreversibility, stability and energy balance. This new formulation is compared with that usually postulated and its merits are emphasized.

Key words: Damage, stability, variational methods

Mots-clés : Endommagement, stabilité, méthodes variationnelles

\section{Abridged English version}

The first stage in the construction of a non local damage model consists in introducing the gradient of damage $\nabla \alpha$ as a local state variable and hence as a variable of the strain work function $W$. Accordingly, in an isotropic and linearized setting, the strain work function simply differs from its non regularized analog $W_{0}$ by a quadratic term in $\nabla \alpha$, see (2) and (7). Moreover, it is always possible by a change of the damage variable to assume that the new variable varies in the interval $[0,1]$ and that the multiplicative factor of $\nabla \alpha \cdot \nabla \alpha$ is a positive constant. The damage model is then perfectly defined by the two functions $\hat{d}, \hat{\psi}$ and the constant $\hat{\gamma}$, see (9). A typical example used as a regularization of the Griffith brittle fracture model in [1] is given by (10).

Email addresses: pham@lmm.jussieu.fr (Kim Pham), marigo@lms.polytechnique.fr (corresponding author) (Jean-Jacques Marigo). 
The second stage consists in formulating the damage evolution problem in terms of the three physical principles of irreversibility, stability and energy balance introduced in [6] for local damage models. Specifically, let us define the set $\mathcal{C}_{t}$ of admissible displacement fields at time $t$ as the affine space $U_{t}+\mathcal{C}^{0}$ where $\mathcal{C}^{0}$ is a linear space and $U_{t}$ is a given displacement field. Let $\mathcal{D}_{0}=\left\{D \in H^{1}(\Omega,[0,1]): D=0\right.$ on $\left.\partial^{e} \Omega\right\}$ be the convex set of admissible damage fields where $\partial^{e} \Omega$ denotes the part of the boundary of the body $\Omega$ where the damage is fixed at 0 . The energy of the body at time $t$ in the admissible state $\left(u^{*}, D^{*}\right)$ is defined by (11) where $W_{t}^{e}$ denotes the potential of the applied forces. Then the evolution problem consists in finding, for all $t \geq 0$, the displacement and damage fields $\left(u_{t}, D_{t}\right)$ which satisfy the three following items:

(IR) $\quad t \mapsto D_{t}$ must increase from $D_{0}$;

(ST) $\quad\left(u_{t}, D_{t}\right)$ must be directionally-stable in the sense of [6, Definition 3.3]:

$\forall\left(u^{*}, D^{*}\right) \in \mathcal{C}_{t} \times \mathcal{D}\left(D_{t}\right), \quad \exists \bar{h}>0, \quad \forall h \in[0, \bar{h}], \quad \hat{\mathcal{P}}_{t}\left(u_{t}+h\left(u^{*}-u_{t}\right), D_{t}+h\left(D^{*}-D_{t}\right)\right) \geq \hat{\mathcal{P}}_{t}\left(u_{t}, D_{t}\right) ;$

(EB) The balance of energy must be satisfied, cf [6, Definition 3.4]:

$$
\hat{\mathcal{P}}_{t}\left(u_{t}, D_{t}\right)=\hat{\mathcal{P}}_{0}\left(u_{0}, D_{0}\right)+\int_{0}^{t}\left(\int_{\Omega} \sigma_{t^{\prime}} \cdot \varepsilon\left(\dot{U}_{t^{\prime}}\right) d x-W_{t^{\prime}}^{e}\left(\dot{U}_{t^{\prime}}\right)-\dot{W}_{t^{\prime}}^{e}\left(u_{t^{\prime}}\right)\right) d t^{\prime} .
$$

In (ST), $\mathcal{D}\left(D_{t}\right)=\left\{D^{*} \in \mathcal{D}_{0}: D^{*} \geq D_{t}\right\}$ denotes the set of damage states which are accessible from $D_{t}$, whereas in (EB), $\dot{U}$ and $\dot{W}^{e}$ denote the rate of the loading data and $\sigma$ is the stress field.

Finally, this variational formulation of the evolution problem is compared to what is usually postulated in the literature. Assuming that there exists a solution, smooth in space and time, we first show that $\left(u_{t}, D_{t}\right)$ must satisfy the equilibrium equations and the following Kuhn-Tucker conditions:

$$
\begin{gathered}
\frac{\partial \hat{W}_{0}}{\partial D}\left(\varepsilon\left(u_{t}\right), D_{t}\right)-\operatorname{div}\left(\hat{\gamma} \operatorname{grad} D_{t}\right) \geq 0 \quad \text { in } \quad \Omega_{t}^{d}, \quad \hat{\gamma} \frac{\partial D_{t}}{\partial n} \geq 0 \quad \text { on } \quad \partial_{t}^{d} \Omega \\
\dot{D}_{t}\left(\frac{\partial \hat{W}_{0}}{\partial D}\left(\varepsilon\left(u_{t}\right), D_{t}\right)-\operatorname{div}\left(\hat{\gamma} \operatorname{grad} D_{t}\right)\right)=0 \quad \text { in } \quad \Omega_{t}^{d}, \quad \dot{D}_{t} \hat{\gamma} \frac{\partial D_{t}}{\partial n}=0 \quad \text { on } \quad \partial_{t}^{d} \Omega
\end{gathered}
$$

where $\Omega_{t}^{d}$ denotes the part of the body where the damage is less than 1 and $\partial_{t}^{d} \Omega$ denotes the part of the boundary where the damage is not fixed at 0 and less than 1.

However these conditions are only sufficient for hardening materials. In the case of softening materials, $\left(u_{t}, D_{t}\right)$ must also satisfy second order stability conditions. In the case where the elastic potential is quadratic in $\varepsilon$, i.e. $\hat{\psi}(\varepsilon, D)=\frac{1}{2} \hat{A}(D) \varepsilon \cdot \varepsilon$, these additional conditions can be set in terms of the minimum of the Rayleigh ratio $\mathcal{R}_{t}$ over $\mathcal{C}^{0} \times \mathcal{D}_{t}^{a}$ where

$$
\mathcal{R}_{t}(\bar{u}, \bar{D})=\frac{\int_{\Omega_{t}^{d}} \hat{\mathrm{A}}\left(D_{t}\right)\left(\varepsilon(\bar{u})-\bar{D} \hat{\mathrm{S}}^{\prime}\left(D_{t}\right) \sigma_{t}\right) \cdot\left(\varepsilon(\bar{u})-\bar{D} \hat{\mathrm{S}}^{\prime}\left(D_{t}\right) \sigma_{t}\right) d x+\int_{\Omega_{t}^{a}} \hat{\gamma} \nabla \bar{D} \cdot \nabla \bar{D} d x}{\int_{\Omega_{t}^{a}}\left(\frac{1}{2} \hat{\mathrm{S}}^{\prime \prime}\left(D_{t}\right) \sigma_{t} \cdot \sigma_{t}-\hat{d}^{\prime \prime}\left(D_{t}\right)\right) \bar{D}^{2} d x}
$$

and

$$
\mathcal{D}_{t}^{a}=\left\{\bar{D} \in H^{1}(\Omega): \bar{D} \geq 0, \bar{D}=0 \text { in } \Omega \backslash \Omega_{t}^{a}, \bar{D}=0 \text { on } \partial \Omega \backslash \partial_{t}^{a} \Omega\right\},
$$

$\hat{\mathrm{S}}(D)=\hat{\mathrm{A}}(D)^{-1}$ denoting the damaged compliance tensor whereas

$$
\Omega_{t}^{a}=\left\{x \in \Omega_{t}^{d}: \frac{\partial \hat{W}_{0}}{\partial D}\left(\varepsilon\left(u_{t}\right), D_{t}\right)-\operatorname{div}\left(\hat{\gamma} \operatorname{grad} D_{t}\right)=0\right\}, \quad \partial_{t}^{a} \Omega=\left\{x \in \partial_{t}^{d} \Omega: \frac{\partial D_{t}}{\partial n}=0\right\} .
$$

Specifically, we show that, besides the previous conditions, it is necessary (resp. sufficient) that $\min _{\mathcal{C}^{0} \times \mathcal{D}_{t}^{a}} \mathcal{R}_{t} \geq$ 1 (resp. $>1$ ) for every $t$.

\section{Introduction}

Il s'agit de fournir un cadre général pour l'écriture de lois d'endommagement à gradient. Il s'avère que les concepts introduits dans la première partie [6] pour construire des modèles d'endommagement locaux 
sont directement extensibles aux modèles non locaux de ce type. Il suffit en fait de changer la forme du travail de déformation en y incluant des termes en gradient d'endommagement, ce que nous nous proposons de faire dans le cadre d'une théorie linéarisée pour un matériau isotrope. Sinon, le problème d'évolution reste formellement le même et s'écrit toujours en termes des trois principes d'irréversibilité, de stabilité et de conservation de l'énergie. Nous nous proposons ensuite de comparer le modèle ainsi construit avec les modèles existants ce qui nous permettra de faire ressortir tous les intérêts de l'approche variationnelle.

\section{La construction des modèles}

2.1. Le modèle local sous-jacent. Dans l'approche variationnelle, la construction du modèle non-local part du modèle local sous-jacent dont on rappelle ici les principaux aspects, cf [6].

(i) l'état d'endommagement est décrit à l'aide d'un scalaire $\alpha$ croissant de 0 à $\alpha_{m}$;

(ii) à $\alpha$ fixé, le matériau a un comportement élastique. Son élasticité, dépendante de $\alpha$, est caractérisée par le potentiel élastique $\psi(\varepsilon, \alpha)$ où $\psi$ est une fonction strictement convexe, homogène de degré 2 en $\varepsilon$, à $\alpha$ fixé. De plus $\psi$ est continûment différentiable. On traduit que le matériau s'assouplit quand l'endommagement augmente en demandant que $\psi$ soit une fonction décroissante de $\alpha$. D'où

$$
\sigma=\frac{\partial \psi}{\partial \varepsilon}(\varepsilon, \alpha), \quad \psi(k \varepsilon, \alpha)=k^{2} \psi(\varepsilon, \alpha), \quad \frac{\partial \psi}{\partial \alpha}(\varepsilon, \alpha) \leq 0, \quad \forall k \geq 0, \forall(\varepsilon, \alpha) \in \mathbb{M}_{s}^{n} \times\left[0, \alpha_{m}\right)
$$

où $\sigma$ et $\varepsilon$ représentent l'état local des contraintes et des déformations, $\mathbb{M}_{s}^{n}$ désigne l'ensemble des matrices $n \times n$ symétriques, $n$ étant la dimension de l'espace;

(iii) pour des raisons d'irréversibilité, l'endommagement ne peut que croître. Son évolution est régie par une loi à seuil. Dans le cas de modèles locaux, grâce au postulat de Drucker-Ilyushin, on peut montrer que le travail de déformation $W_{0}$ est une fonction de l'état local $(\varepsilon, \alpha)$ qui s'écrit

$$
W_{0}(\varepsilon, \alpha)=\psi(\varepsilon, \alpha)+d(\alpha)
$$

où $d(\alpha)$ représente l'énergie dissipée lorsque l'on fait passer l'état local du matériau de $(0,0)$ à $(\varepsilon, \alpha)$. La fonction $\alpha \mapsto d(\alpha)$ est continûment différentiable, nulle en 0 et strictement croissante :

$$
d(0)=0, \quad d^{\prime}(\alpha)>0 \quad \forall \alpha \in\left[0, \alpha_{m}\right) .
$$

La loi d'évolution de $\alpha$ est une loi standard qui s'écrit localement

$$
\dot{\alpha} \geq 0, \quad \frac{\partial W_{0}}{\partial \alpha}(\varepsilon, \alpha) \geq 0, \quad \dot{\alpha} \frac{\partial W_{0}}{\partial \alpha}(\varepsilon, \alpha)=0 .
$$

2.2. La nouvelle forme du travail de déformation. Le gradient d'endommagement $\nabla \alpha$ est maintenant une variable d'état local dont dépend le travail de déformation qui devient donc la fonction $W: \mathbb{M}_{s}^{n} \times$ $\left[0, \alpha_{m}\right] \times \mathbb{R}^{n} \rightarrow \mathbb{R},(\varepsilon, \alpha, \nabla \alpha) \mapsto W(\varepsilon, \alpha, \nabla \alpha)$. Dans le cadre d'une théorie linéarisée, on développe $W$ au voisinage de "l'état d'équilibre thermodynamique" $(0, \alpha, 0)$ jusquà l'ordre 2 en $\varepsilon$ et $\nabla \alpha$. Cette linéarisation n'est que partielle puisqu'on ne peut pas linéariser en $\alpha$ à cause des fortes variations de la rigidité. Du fait de l'asymétrie envisagée entre traction et compression, $W$ est une fois continûment différentiable (ce qui permet d'avoir une relation contrainte-déformation continue), mais pas deux fois. Les termes du premier ordre en $\varepsilon$ sont donc linéaires, alors que les termes du second ordre en $\varepsilon$ sont seulement homogènes de degré 2. On a donc

$$
W(\varepsilon, \alpha, \nabla \alpha)=d(\alpha)+\sigma_{0}(\alpha) \cdot \varepsilon+\lambda(\alpha) \cdot \nabla \alpha+\psi(\varepsilon, \alpha)+\Lambda(\alpha) \cdot(\varepsilon \otimes \nabla \alpha)+\Gamma(\alpha) \nabla \alpha \cdot \nabla \alpha
$$

où le point désigne le produit scalaire entre vecteurs ou tenseurs du même ordre. Dans (5) apparaissent les termes "non locaux" $\lambda(\alpha) \in \mathbb{R}^{n}, \Lambda(\alpha) \in \mathbb{M}_{s}^{n} \otimes \mathbb{R}^{n}$ et $\Gamma(\alpha) \in \mathbb{M}_{s}^{n}$ alors que $\sigma_{0}(\alpha)$ représente une précontrainte variant avec l'endommagement que nous supposerons nulle comme nous l'avions fait dans le modèle local. Les termes $d(\alpha)$ et $\psi(\varepsilon, \alpha)$ sont ceux du modèle local, $\psi$ étant homogène de degré 2 en $\varepsilon$. On pourrait aussi envisager des développements non réguliers en $\nabla \alpha$ mais on opte ici pour le choix le plus simple. 
Si nous supposons que le matériau est isotrope et que la variable d'endommagement est un scalaire objectif (i.e. invariant dans tout changement de référentiel), $W$ doit alors satisfaire les conditions d'invariance

$$
W\left(Q \varepsilon Q^{T}, \alpha, Q \nabla \alpha\right)=W(\varepsilon, \alpha, \nabla \alpha), \quad \forall Q \in \mathbb{O}^{n}, \quad \forall(\varepsilon, \alpha, \nabla \alpha) \in \mathbb{M}_{s}^{n} \times\left[0, \alpha_{m}\right] \times \mathbb{R}^{n}
$$

où $\mathbb{O}^{n}$ désigne le groupe orthogonal. Dans ce cas le vecteur $\lambda(\alpha)$ et le tenseur d'ordre trois $\Lambda(\alpha)$ sont nécessairement nuls, alors que le tenseur d'ordre deux $\Gamma(\alpha)$ est proportionnel à l'identité. Pour des raisons évidentes, si l'on veut que les termes non locaux soient régularisants, il faut que $\Gamma(\alpha)$ soit défini positif, ce qui permet d'écrire $\Gamma(\alpha)=\gamma(\alpha) \mathbf{I}$ avec $\gamma(\alpha)>0$.

En résumé, pour un matériau isotrope, en théorie linéarisée et en supposant qu'il n'y a pas de précontrainte, le travail de déformation s'écrit

$$
W(\varepsilon, \alpha, \nabla \alpha)=d(\alpha)+\psi(\varepsilon, \alpha)+\gamma(\alpha) \nabla \alpha \cdot \nabla \alpha
$$

avec $\psi\left(Q \varepsilon Q^{T}, \alpha\right)=\psi(\varepsilon, \alpha)$. On voit qu'il ne diffère de son homologue local (2) que par l'ajout d'un terme quadratique en $\nabla \alpha$ dont on attend qu'il ait des vertus régularisantes. Notons que cette introduction purement phénoménologique de termes en gradient d'endommagement dans l'énergie souffre d'un manque de base micro-mécanique. Alors qu'il est facile de justifier les termes "locaux" par des arguments basés sur le comportement effectif de matériau micro-fissuré, il n'existe pas à ce jour, à notre connaissance, de telle justification pour les termes non locaux.

2.3. Normalisation de la variable d'endommagement. À ce stade la variable d'endommagement a été choisie arbitrairement, elle ne sert qu'à paramétrer l'évolution des propriétés thermomécaniques du matériau. On peut en changer, via un changement de variable, sans changer de modèle. Une infinité de choix sont possibles. Un choix particulièrement intéressant consiste à prendre pour variable d'endommagement l'énergie volumique dissipée, ce qui revient à faire le changement de variable $\alpha \mapsto d=d(\alpha)$. Cela permet d'exprimer simplement les propriétés d'écrouissage du matériau, cf [6, Proposition 2.3]. Avec ce choix, le travail de déformation s'écrit

$$
\tilde{W}(\varepsilon, d, \nabla d)=d+\tilde{\psi}(\varepsilon, d)+\tilde{\gamma}(d) \nabla d \cdot \nabla d .
$$

Mais un autre choix tout aussi intéressant en pratique consiste à faire un changement de variable de façon à faire apparaître une constante devant le terme quadratique non local au lieu de la fonction $\gamma(\alpha)$. Il est même autorisé de prendre cette constante égale à 1 . Il suffit pour cela de faire le changement de variable $\alpha \mapsto \Delta=\Delta(\alpha):=\int_{0}^{\alpha} \sqrt{\gamma(\beta)} d \beta$. Toutefois, pour comparer différents matériaux entre eux, on peut avoir intérêt à normaliser la variable d'endommagement en la prenant sans dimension et variant par exemple entre 0 et 1 lorsque $\alpha$ varie entre 0 et $\alpha_{m}$. Ainsi, en supposant que $\Delta\left(\alpha_{m}\right)<+\infty$ et en faisant le changement de variable $\alpha \mapsto D=\Delta(\alpha) / \Delta\left(\alpha_{m}\right)$, le travail de déformation peut s'écrire

$$
\hat{W}(\varepsilon, D, \nabla D)=\hat{d}(D)+\hat{\psi}(\varepsilon, D)+\frac{\hat{\gamma}}{2} \nabla D \cdot \nabla D,
$$

où la variable $D$ varie entre 0 et 1 et $\hat{\gamma}>0$ est maintenant une constante caractéristique du matériau qui a la dimension d'une force. Cette constante pourrait aussi s'écrire $\hat{\gamma}=E_{0} \ell^{2}, E_{0}$ désignant le module d'Young du matériau sain et $\ell>0$ étant donc une longueur caractéristique du matériau. Avec ce choix, sachant que la loi d'évolution de l'endommagement se formule entièrement en terme du travail de déformation, le comportement du matériau est caractérisé par les fonctions $\hat{d}, \hat{\psi}$ et la constante $\hat{\gamma}$. Il est à noter que les propriétés de monotonie, d'homogénéité et d'isotropie postulées pour les fonctions $d$ et $\psi$ ne sont pas affectées par le changement de variable et sont encore vérifiées par $\hat{d}$ et $\hat{\psi}$.

2.4. Exemple. On peut transformer n'importe quel modèle local en un modèle non local en rajoutant simplement le terme quadratique en gradient d'endommagement. Notons toutefois que si on a normalisé le modèle local, alors on doit a priori introduire une fonction $\gamma(\alpha)$ comme facteur multiplicatif du terme non local. À titre d'exemple, reprenons le modèle 3D introduit dans [6] et normalisons-le de façon à faire apparaître une constante dans le terme en gradient. Il s'écrit alors

$$
\hat{W}(\varepsilon, D, \nabla D)=\hat{d}(D)+\frac{1}{2} \hat{K}(D)\left(\operatorname{Tr} \varepsilon^{+}\right)^{2}+\frac{1}{2} K_{0}\left(\operatorname{Tr} \varepsilon^{-}\right)^{2}+\hat{\mu}(D) \varepsilon^{D} \cdot \varepsilon^{D}+\frac{\hat{\gamma}}{2} \nabla D \cdot \nabla D
$$

où $\hat{K}(D)$ et $\hat{\mu}(D)$ représentent le module de compressibilité et le module de cisaillement du matériau endommagé, $K_{0}$ est le module de compressibilité du matériau sain, $\varepsilon^{D}$ est le déviateur de $\varepsilon, \operatorname{Tr} \varepsilon$ sa trace, 
$\operatorname{Tr} \varepsilon^{ \pm}=\max \{0, \pm \operatorname{Tr} \varepsilon\}$. L'identification de ce modèle passe donc par la connaissance des trois fonctions $\hat{d}, \hat{K}, \hat{\mu}$ et de la constante $\hat{\gamma}$. Ce type de modèle est utilisé comme régularisation du modèle de rupture de Griffith, cf [1].

\section{Le problème d'évolution de l'endommagement}

3.1. Sa formulation. Du fait du caractère non local du modèle, la loi d'évolution doit se formuler directement au niveau de la structure. On considère une structure dont la configuration de référence naturelle est l'ouvert $\Omega$ de $\mathbb{R}^{n}$, qui est constituée d'un (plusieurs) matériau(x) endommageable(s) du type précédent dont le paramètre d'endommagement a été normalisé. Les fonctions d'état sont surmontées d'un ^et, dans le cas hétérogène, les fonctions $\hat{d}, \hat{\psi}$ et le scalaire $\hat{\gamma}$ dépendent de $x$. Cette structure est dans un état d'endommagement donné $D_{0}$ à l'instant 0 avant d'être soumise à un chargement dépendant d'un paramètre $t \geq 0$. Le problème d'évolution quasi-statique consiste à chercher, à chaque "instant" $t$, les champs des déplacements $u_{t}$ et d'endommagement $D_{t}$. Nous le formulons en reprenant les 3 items d'irréversibilité, de stabilité et de conservation de l'énergie introduits dans [6] pour les modèles locaux. Cela passe tout d'abord par l'adaptation de quelques définitions et par l'introduction de quelques hypothèses sur le chargement.

(i) Nous supposons que l'ensemble des déplacements cinématiquement admissibles à l'instant $t$ est un espace affine de la forme $\mathcal{C}_{t}=U_{t}+\mathcal{C}^{0}$ où $\mathcal{C}^{0}$ est un espace vectoriel indépendant du temps et $U_{t}$ est un champ de déplacement donné dépendant régulièrement de $t$.

(ii) La structure est soumise à un système de forces dépendant régulièrement du temps et dont le potentiel à l'instant $t$ est la forme linéaire $W_{t}^{e}$.

(iii) Du point de vue de la régularité, pour que l'énergie de la structure dans un état d'endommagement $D$ soit finie, il faut que $D$ soit dans $H^{1}(\Omega,[0,1])$. De ce fait, il admet une trace sur le bord du domaine et il est tout à fait licite d'imposer des conditions aux limites à $D$ sur toute ou partie de la frontière. Sur le plan théorique, n'importe quel type de conditions aux limites pour $D$ est envisageable. D'un point de vue physique, on peut imaginer des systèmes de fixation de la structure sur une partie du bord qui feraient que $D$ doit rester nul sur ce bord. Pour simplifier, nous supposons ici que $D=0$ sur la partie (indépendante du temps) $\partial^{e} \Omega$ du bord et l'ensemble convexe (indépendant du temps) des endommagements admissibles est donc $\mathcal{D}_{0}=\left\{D \in H^{1}(\Omega,[0,1]): D=0\right.$ sur $\left.\partial^{e} \Omega\right\}$.

(iv) À un couple $\left(u^{*}, D^{*}\right) \in \mathcal{C}_{t} \times \mathcal{D}_{0}$ admissible à l'instant $t$ on associe l'énergie totale $\hat{\mathcal{P}}_{t}\left(u^{*}, D^{*}\right)$ de la structure dans cet état

$$
\hat{\mathcal{P}}_{t}\left(u^{*}, D^{*}\right)=\int_{\Omega} \hat{W}\left(x, \varepsilon\left(u^{*}\right)(x), D^{*}(x), \nabla D^{*}(x)\right) d x-W_{t}^{e}\left(u^{*}\right)
$$

où $\varepsilon\left(u^{*}\right)$ désigne la partie symétrique du gradient de $u^{*}$.

(v) À $D \in \mathcal{D}_{0}$ on associe l'ensemble $\mathcal{D}(D)$ des états d'endommagement accessibles depuis $D$, qui sont restreints du fait de la condition d'irréversibilité : $\mathcal{D}(D)=\left\{D^{*} \in \mathcal{D}_{0}: D^{*} \geq D\right\}$.

Munis de ces hypothèses et définitions, nous sommes en mesure de formuler le problème d'évolution en reprenant simplement les trois principes d'irréversibilité, de stabilité et de bilan d'énergie introduits dans [6]. Cela conduit à la

Définition 3.1 Le problème d'évolution consiste à trouver pour tout $t \geq 0,\left(u_{t}, D_{t}\right) \in \mathcal{C}_{t} \times \mathcal{D}_{0}$ tel que

(IR) $t \mapsto D_{t}$ soit croissant depuis $D_{0}$;

(ST) $\left(u_{t}, D_{t}\right)$ soit stable au sens suivant, cf [6, Définition 3.3] :

$\forall\left(u^{*}, D^{*}\right) \in \mathcal{C}_{t} \times \mathcal{D}\left(D_{t}\right), \quad \exists \bar{h}>0, \quad \forall h \in[0, \bar{h}], \quad \hat{\mathcal{P}}_{t}\left(u_{t}+h\left(u^{*}-u_{t}\right), D_{t}+h\left(D^{*}-D_{t}\right)\right) \geq \hat{\mathcal{P}}_{t}\left(u_{t}, D_{t}\right) ;$

(EB) Le bilan d'énergie suivant soit satisfait à chaque instant, cf [6, Définition 3.4] :

$$
\hat{\mathcal{P}}_{t}\left(u_{t}, D_{t}\right)=\hat{\mathcal{P}}_{0}\left(u_{0}, D_{0}\right)+\int_{0}^{t}\left(\int_{\Omega} \sigma_{t^{\prime}} \cdot \varepsilon\left(\dot{U}_{t^{\prime}}\right) d x-W_{t^{\prime}}^{e}\left(\dot{U}_{t^{\prime}}\right)-\dot{W}_{t^{\prime}}^{e}\left(u_{t^{\prime}}\right)\right) d t^{\prime} .
$$


Dans le bilan d'énergie, $u_{0}$ est obtenu en résolvant le problème d'élasticité linéaire associé, i.e. $u_{0}=$ $\operatorname{argmin}_{v \in \mathcal{C}_{0}} \hat{\mathcal{P}}_{0}\left(v, D_{0}\right), \sigma_{t}$ désigne le champ de contraintes régnant à l'instant $t, \sigma_{t}=\frac{\partial \hat{\psi}}{\partial \varepsilon}\left(\varepsilon\left(u_{t}\right), D_{t}\right)$, alors que $\dot{U}_{t}$ et $\dot{W}_{t}^{e}$ représentent les vitesses des données de chargement.

On voit donc que le problème d'évolution est formellement le même que celui proposé pour les modèles locaux. Mais, évidemment du fait de la présence de termes non locaux dans l'énergie, ces deux problèmes n'admettent pas en général les mêmes solutions. Notons que les propriétés d'irréversibilité, de stabilité et de conservation de l'énergie sont invariantes par changement de la variable d'endommagement. Si elles sont vérifiées par le modèle normalisé, alors elles le sont aussi par tout représentant du même modèle.

D'un point de vue numérique, un algorithme naturel découlant de cette formulation variationnelle est l'algorithme dit de minimisation alternée qui consiste à minimiser par rapport à $u$ à $D$ fixé, puis par rapport à $D$ à $u$ fixé et d'itérer jusqu'à convergence. C'est un algorithme de descente qui converge (mais pas forcément vers un état stable), cf [3] pour des détails et [3,1] pour des exemples.

3.2. Comparaison avec les formulations usuelles. Supposons que le problème d'évolution admette une solution régulière en temps et en espace et raisonnons par conditions nécessaires. En divisant l'inégalité de stabilité (ST) par $h>0$ et en passant à la limite quand $h \rightarrow 0$, on en déduit les conditions nécessaires de stabilité dites d'ordre 1 que doit vérifier $\left(u_{t}, D_{t}\right)$ à l'instant $t$ :

$$
\hat{\mathcal{P}}_{t}^{\prime}\left(u_{t}, D_{t}\right)\left(u^{*}-u_{t}, D^{*}-D_{t}\right) \geq 0, \quad \forall\left(u^{*}, D^{*}\right) \in \mathcal{C}_{t} \times \mathcal{D}\left(D_{t}\right)
$$

où $\hat{\mathcal{P}}_{t}^{\prime}\left(u_{t}, D_{t}\right)$ désigne la dérivée de Gâteaux en $\left(u_{t}, D_{t}\right)$, i.e. la forme linéaire définie par

$$
\hat{\mathcal{P}}_{t}^{\prime}\left(u_{t}, D_{t}\right)(\bar{u}, \bar{D})=\int_{\Omega} \sigma_{t} \cdot \varepsilon(\bar{u}) d x-W_{t}^{e}(\bar{u})+\int_{\Omega}\left(\frac{\partial \hat{W}_{0}}{\partial D}\left(\varepsilon\left(u_{t}\right), D_{t}\right) \bar{D}+\hat{\gamma} \nabla D_{t} \cdot \nabla \bar{D}\right) d x .
$$

En prenant $D^{*}=D_{t}$ dans $(12)$ et en remarquant que $\mathcal{C}_{t}$ est un espace affine, on retrouve la formulation variationnelle de l'équilibre, i.e.

$$
\int_{\Omega} \sigma_{t} \cdot \varepsilon(\bar{u}) d x=W_{t}^{e}(\bar{u}), \quad \forall \bar{u} \in \mathcal{C}^{0} .
$$

En reportant dans (12) on obtient la formulation variationnelle du critère d'endommagement non local, à savoir

$$
\int_{\Omega}\left(\frac{\partial \hat{W}_{0}}{\partial D}\left(\varepsilon\left(u_{t}\right), D_{t}\right)\left(D^{*}-D_{t}\right)+\hat{\gamma} \nabla D_{t} \cdot \nabla\left(D^{*}-D_{t}\right)\right) d x \geq 0, \quad \forall D^{*} \in \mathcal{D}\left(D_{t}\right)
$$

Notons $\Omega_{t}^{c}$ le domaine où $D_{t}=1$ (ce domaine peut encore contenir de l'énergie élastique si la perte de rigidité n'est que partielle quand $D=1$ ) et $\Omega_{t}^{d}$ celui où $0 \leq D_{t}<1$. Le cas où $\Omega_{t}^{c}=\Omega$ est trivial, supposons donc que $\Omega_{t}^{d} \neq \emptyset$. Comme $D^{*}=D_{t}=1$ dans $\Omega_{t}^{c}$, l'intégrale dans (14) ne porte que sur $\Omega_{t}^{d}$. On peut également partitionner $\partial \Omega$ en trois parties (dont certaines peuvent être vides) : (i) $\partial^{e} \Omega$ où $D_{t}=D^{*}=0$; (ii) $\partial_{t}^{d} \Omega$ où $0 \leq D_{t} \leq D^{*} \leq 1$ et $D_{t}<1$; (iii) $\partial_{t}^{c} \Omega$ où $D_{t}=D^{*}=1$. En vertu des propriétés de la trace pour des fonctions de $H^{1}(\Omega)$, on a $D_{t}=D^{*}=1$ sur $\partial \Omega_{t}^{c}$ et donc $\partial^{e} \Omega \subset \partial \Omega_{t}^{d}$ ainsi que $\partial_{t}^{d} \Omega \subset \partial \Omega_{t}^{d}$. Après une intégration par parties dans $(14)$, il vient, $\forall D^{*} \in \mathcal{D}\left(D_{t}\right)$ :

$$
\int_{\Omega_{t}^{d}}\left(\frac{\partial \hat{W}_{0}}{\partial D}\left(\varepsilon\left(u_{t}\right), D_{t}\right)-\operatorname{div}\left(\hat{\gamma} \operatorname{grad} D_{t}\right)\right)\left(D^{*}-D_{t}\right) d x+\int_{\partial_{t}^{d} \Omega} \hat{\gamma} \frac{\partial D_{t}}{\partial n}\left(D^{*}-D_{t}\right) d S \geq 0
$$

où $n$ désigne la normale unitaire sortante à $\Omega$. Sous réserve de régularité suffisante des champs, on obtient par un raisonnement classique de Calcul des Variations les conditions locales suivantes

$$
\frac{\partial \hat{W}_{0}}{\partial D}\left(\varepsilon\left(u_{t}\right), D_{t}\right)-\operatorname{div}\left(\hat{\gamma} \operatorname{grad} D_{t}\right) \geq 0 \quad \text { dans } \quad \Omega_{t}^{d}, \quad \hat{\gamma} \frac{\partial D_{t}}{\partial n} \geq 0 \quad \text { sur } \quad \partial_{t}^{d} \Omega
$$

qui constituent donc l'écriture locale du critère d'endommagement pour un modèle à gradient. Elles sont le pendant du critère portant sur le taux de restitution d'énergie pour une loi locale, cf (4). Ces inégalités sont conformes à ce qui est généralement postulé dans la littérature, cf [5], [4]. Mais notons qu'elles découlent ici de la condition de stabilité. Il serait donc facile de les généraliser à des modèles plus complexes (matériaux anisotropes ou non linéaires par exemple). Remarquons aussi qu'elles fournissent automatiquement des conditions aux limites naturelles sur les bords du domaine où l'endommagement 
peut encore évoluer ou aux interfaces entre différents matériaux. En effet, dans ce dernier cas, on obtient directement par l'approche variationnelle que $\hat{\gamma} \frac{\partial D_{t}}{\partial n}$ doit être continu sur toute surface incluse dans $\Omega_{t}^{d}$. C'est implicitement contenu dans la première inégalité de (16) si on la lit au sens des distributions.

Exploitons maintenant le bilan d'énergie pour essayer de récupérer la condition dite de cohérence, i.e. le pendant de l'égalité $\dot{\alpha} \frac{\partial W}{\partial \alpha}(\varepsilon, \alpha)=0$ du modèle local. Supposons toujours la régularité en temps et en espace de la solution et dérivons (EB) par rapport à $t$. Il vient

$$
\begin{aligned}
0 & =\frac{d}{d t} \hat{\mathcal{P}}_{t}\left(u_{t}, D_{t}\right)-\int_{\Omega} \sigma_{t} \cdot \varepsilon\left(\dot{U}_{t}\right) d x+W_{t}^{e}\left(\dot{U}_{t}\right)+\dot{W}_{t}^{e}\left(u_{t}\right) \\
& =\int_{\Omega} \sigma_{t} \cdot \varepsilon\left(\dot{u}_{t}-\dot{U}_{t}\right) d x-W_{t}^{e}\left(\dot{u}_{t}-\dot{U}_{t}\right)+\int_{\Omega}\left(\frac{\partial \hat{W}_{0}}{\partial D}\left(\varepsilon\left(u_{t}\right), D_{t}\right) \dot{D}_{t}+\hat{\gamma} \nabla D_{t} \cdot \nabla \dot{D}_{t}\right) d x \\
& =\int_{\Omega}\left(\frac{\partial \hat{W}_{0}}{\partial D}\left(\varepsilon\left(u_{t}\right), D_{t}\right) \dot{D}_{t}+\hat{\gamma} \nabla D_{t} \cdot \nabla \dot{D}_{t}\right) d x
\end{aligned}
$$

les deux premiers termes de la deuxième ligne se compensant grâce à l'équilibre (13). En intégrant par parties le terme en gradient, comme $\dot{D}_{t}=0$ dans $\Omega_{t}^{c}$, sur $\partial^{e} \Omega$ et sur $\partial_{t}^{c} \Omega$, on obtient

$$
0=\int_{\Omega_{t}^{d}}\left(\frac{\partial \hat{W}_{0}}{\partial D}\left(\varepsilon\left(u_{t}\right), D_{t}\right)-\operatorname{div}\left(\hat{\gamma} \operatorname{grad} D_{t}\right)\right) \dot{D}_{t} d x+\int_{\partial_{t}^{d} \Omega} \hat{\gamma} \frac{\partial D_{t}}{\partial n} \dot{D}_{t} d S
$$

Il suffit de tenir compte de l'irréversibilité qui exige que $\dot{D}_{t} \geq 0$ et du critère d'endommagement (16) pour obtenir la condition de cohérence désirée

$$
\dot{D}_{t}\left(\frac{\partial \hat{W}_{0}}{\partial D}\left(\varepsilon\left(u_{t}\right), D_{t}\right)-\operatorname{div}\left(\hat{\gamma} \operatorname{grad} D_{t}\right)\right)=0 \quad \text { dans } \quad \Omega_{t}^{d}, \quad \dot{D}_{t} \hat{\gamma} \frac{\partial D_{t}}{\partial n}=0 \quad \text { sur } \quad \partial_{t}^{d} \Omega .
$$

Il est à noter que les conditions aux limites naturelles que l'on a obtenues font qu'en général on aura $\partial D / \partial n=0$ sur les parties du bord où $D$ peut évoluer.

En résumé, on vient de montrer que les relations de comportement généralement adoptées pour les modèles à gradient se déduisent dans l'approche variationnelle de la condition d'irréversibilité, de la condition de stabilité d'ordre 1 et du bilan d'énergie. Mais les inégalités (16) ne sont que des conditions nécessaires, en général non suffisantes, pour que la condition de stabilité soit satisfaite. Nous nous proposons dans le prochain paragraphe d'établir des conditions nécessaires supplémentaires. Insistons aussi sur le fait que les conditions (16) et (17) n'ont un sens que dans des évolutions régulières, alors que (IR), (ST) et (EB) valent aussi dans des évolutions non régulières.

3.3. Les conditions de stabilité d'ordre 2. Pour simplifier la présentation, nous supposons que $\hat{\psi}$ est quadratique en $\varepsilon$, i.e. $\hat{\psi}(\varepsilon, D)=\frac{1}{2} \hat{\mathrm{A}}(D) \varepsilon \cdot \varepsilon$ où $\hat{\mathrm{A}}(D)$ est le tenseur de rigidité endommagé $($ avec $\hat{\mathrm{A}}(D)>0$ si $D<1$ et $\hat{\mathrm{A}}(1)=0$ ), et que $\hat{d}$ et $\hat{\mathrm{A}}$ sont deux fois dérivables par rapport à $D$. Considérons un état $\left(u_{t}, D_{t}\right)$ à l'instant $t$ satisfaisant les conditions de stabilité d'ordre 1 (12) et donc (13) et (14). Posons $(\bar{u}, \bar{D})=\left(u^{*}-u_{t}, D^{*}-D_{t}\right)$ et écrivons la condition de stabilité $(\mathrm{ST})$ en développant $\hat{\mathcal{P}}_{t}\left(u_{t}+h \bar{u}, D_{t}+h \bar{D}\right)$ par rapport à $h$ jusqu'à l'ordre 2 . Il vient

$$
0 \leq h \hat{\mathcal{P}}_{t}^{\prime}\left(u_{t}, D_{t}\right)(\bar{u}, \bar{D})+\frac{h^{2}}{2} \hat{\mathcal{P}}_{t}^{\prime \prime}\left(u_{t}, D_{t}\right)(\bar{u}, \bar{D})+o\left(h^{2}\right)
$$

où $\hat{\mathcal{P}}_{t}^{\prime \prime}\left(u_{t}, D_{t}\right)$ désigne la dérivée seconde de l'énergie en $\left(u_{t}, D_{t}\right)$, forme quadratique par rapport à $(\bar{u}, \bar{D})$. En utilisant le tenseur de souplesse du matériau endommagé $\hat{\mathrm{S}}(D)=\hat{\mathrm{A}}(D)^{-1}$ ainsi que ses dérivées par rapport à $D$, la dérivée seconde de l'énergie peut s'écrire

$$
\begin{aligned}
\hat{\mathcal{P}}_{t}^{\prime \prime}\left(u_{t}, D_{t}\right)(\bar{u}, \bar{D})= & \int_{\Omega_{t}^{d}} \hat{\mathrm{A}}\left(D_{t}\right)\left(\varepsilon(\bar{u})-\bar{D} \hat{\mathrm{S}}^{\prime}\left(D_{t}\right) \sigma_{t}\right) \cdot\left(\varepsilon(\bar{u})-\bar{D} \hat{\mathrm{S}}^{\prime}\left(D_{t}\right) \sigma_{t}\right) d x+\int_{\Omega_{t}^{d}} \hat{\gamma} \nabla \bar{D} \cdot \nabla \bar{D} d x \\
& +\int_{\Omega_{t}^{d}}\left(\hat{d}^{\prime \prime}\left(D_{t}\right)-\frac{1}{2} \hat{\mathrm{S}}^{\prime \prime}\left(D_{t}\right) \sigma_{t} \cdot \sigma_{t}\right) \bar{D}^{2} d x
\end{aligned}
$$

où on a utilisé le fait que $\bar{D}=0$ dans $\Omega_{t}^{c}$. D'après (12), le premier terme du membre de droite de (18) est non négatif. S'il est positif, alors, pour $h$ assez petit, l'inégalité sera vérifiée et l'état $\left(u_{t}, D_{t}\right)$ sera 
stable dans la direction $(\bar{u}, \bar{D})$. Par contre, s'il est nul, alors l'état ne sera stable dans la direction $(\bar{u}, \bar{D})$ que si la dérivée seconde est non négative (et il sera stable dans cette direction si la dérivée seconde est positive). Or, en vertu de la positivité de $\hat{A}$ et de $\hat{\gamma}$, les deux premiers termes dans l'expression (19) de la dérivée seconde sont non négatifs. Comme $\hat{d}^{\prime \prime}\left(D_{t}\right)-\frac{1}{2} \hat{\mathrm{S}}^{\prime \prime}\left(D_{t}\right) \sigma_{t} \cdot \sigma_{t}$ est positif si le matériau est durcissant mais négatif si le matériau est adoucissant, of [6], la dérivée seconde est nécessairement positive dans toute direction non nulle si le matériau est durcissant mais son signe peut dépendre de la direction si le matériau est adoucissant. Donc, pour les matériaux durcissants, tout état vérifiant (12) est stable et aucune condition supplémentaire n'est à remplir. La question se pose par contre pour les matériaux adoucissants.

Considérons un matériau adoucissant. D'après (15), la dérivée première de l'énergie est nulle si et seulement si $\bar{D}$ est nul sur la partie du domaine et la partie du bord où il n'y a pas égalité dans (16). Introduisons donc $\Omega_{t}^{a}$ et $\partial_{t}^{a} \Omega$, partie du domaine et partie du bord où le critère d'endommagement est atteint, i.e.

$$
\Omega_{t}^{a}=\left\{x \in \Omega_{t}^{d}: \frac{\partial \hat{W}_{0}}{\partial D}\left(\varepsilon\left(u_{t}\right), D_{t}\right)-\operatorname{div}\left(\hat{\gamma} \operatorname{grad} D_{t}\right)=0\right\}, \quad \partial_{t}^{a} \Omega=\left\{x \in \partial_{t}^{d} \Omega: \frac{\partial D_{t}}{\partial n}=0\right\} .
$$

Envisageons des directions $(\bar{u}, \bar{D}) \in \mathcal{C}^{0} \times \mathcal{D}_{t}^{a}$ avec

$$
\mathcal{D}_{t}^{a}=\left\{\bar{D} \in H^{1}(\Omega): \bar{D} \geq 0, \bar{D}=0 \text { dans } \Omega \backslash \Omega_{t}^{a}, \bar{D}=0 \text { sur } \partial \Omega \backslash \partial_{t}^{a} \Omega\right\} .
$$

La dérivée première de l'énergie étant nulle dans de telles directions, la stabilité de l'état va dépendre du signe de la dérivée seconde. Comme celle-ci est la différence entre 2 formes quadratiques non négatives, cela revient à comparer leur quotient à 1. Introduisons donc le quotient de Rayleigh défini $\operatorname{sur} \mathcal{C}^{0} \times \mathcal{D}_{t}^{a}$ :

$$
\mathcal{R}_{t}(\bar{u}, \bar{D})=\frac{\int_{\Omega_{t}^{d}} \hat{\mathrm{A}}\left(D_{t}\right)\left(\varepsilon(\bar{u})-\bar{D} \hat{\mathrm{S}}^{\prime}\left(D_{t}\right) \sigma_{t}\right) \cdot\left(\varepsilon(\bar{u})-\bar{D} \hat{\mathrm{S}}^{\prime}\left(D_{t}\right) \sigma_{t}\right) d x+\int_{\Omega_{t}^{a}} \hat{\gamma} \nabla \bar{D} \cdot \nabla \bar{D} d x}{\int_{\Omega_{t}^{a}}\left(\frac{1}{2} \hat{\mathrm{S}}^{\prime \prime}\left(D_{t}\right) \sigma_{t} \cdot \sigma_{t}-\hat{d}^{\prime \prime}\left(D_{t}\right)\right) \bar{D}^{2} d x}
$$

avec la convention $\mathcal{R}_{t}(\bar{u}, 0)=+\infty$. L'état $\left(u_{t}, D_{t}\right)$ sera stable ou instable suivant que le minimum du quotient de Rayleigh sur $\mathcal{C}^{0} \times \mathcal{D}_{t}^{a}$ sera supérieur ou inférieur à 1 . Comme $\hat{\gamma}>0$, le terme non local a évidemment un rôle stabilisant. Il est bon de noter que cette condition est nécessairement globale, c'est une propriété structurelle et pas seulement matérielle, cf [2]. On peut donc résumer l'ensemble des résultats de cette section par la

Proposition 3.2 Pour qu'une évolution $t \mapsto\left(u_{t}, D_{t}\right)$ partant de $\left(u_{0}, D_{0}\right)$ et régulière en espace et en temps satisfasse le problème d'évolution $(\mathrm{IR}),(\mathrm{ST})$ et $(\mathrm{EB})$, il faut qu'elle vérifie à chaque instant l'équilibre (13), la condition d'irréversibilité, le critère d'endommagement (16) et la condition de cohérence (17). Dans le cas d'un matériau durcissant, ceci est suffisant. Dans le cas d'un matériau adoucissant, il faut (resp. suffit) qu'en plus le minimum du quotient de Rayleigh soit non inférieur (resp. supérieur) à 1 à chaque instant.

\section{Conclusion et perspectives}

L'approche variationnelle permet une construction systématique de modèles d'endommagement à gradient à partir du modèle local sous-jacent. Dans le cas isotrope et dans le cadre d'une théorie linéarisée, cela se réduit à l'introduction d'un terme supplémentaire dans la forme de l'énergie, quadratique en gradient d'endommagement. Après quoi la loi d'évolution se formule toujours à l'aide d'une condition d'irréversibilité, d'un critère de stabilité et d'un bilan d'énergie. On retrouve ainsi automatiquement le critère d'endommagement contenant des dérivées secondes spatiales du champ d'endommagement ainsi que des conditions aux limites naturelles sur le bord du domaine pour la dérivée normale du champ d'endommagement, ce qui donne une assise conceptuelle claire à ce qui résulte sinon le plus souvent de choix arbitraires. Mais le critère de stabilité fait également apparaître des conditions supplémentaires pour les modèles avec adoucissement. Leur étude approfondie fera l'objet des publications suivantes. 


\section{Références}

[1] H. Amor, J.-J. Marigo, and C. Maurini. Variational approach to brittle fracture with unilateral contact : numerical experiments. J. Mech. Phys. Solids, $57: 1209-1229,2009$.

[2] A. Benallal and J.-J. Marigo. Bifurcation and stability issues in gradient theories with softening. Modelling Simul. Mater. Sci. Eng., 15 :283-295, 2007.

[3] B. Bourdin, G. Francfort, and J.-J. Marigo. The variational approach to fracture. J. Elasticity, 91 :5-148, 2008.

[4] C. Comi. Computational modelling of gradient-enhanced damage in quasi-brittle materials. Mech. Cohes.-Frict. Mater., 4(1) :17-36, 1999.

[5] E. Lorentz and S. Andrieux. Analysis of non-local models through energetic formulations. Int. J. Solids Struct., 40 :29052936, 2003.

[6] K. Pham and J.-J. Marigo. L'approche variationnelle de l'endommagement : I. Les concepts fondamentaux. Comptes Rendus Mécanique, à paraître, 2010. 